\title{
Atrial Fibrillation: The Role of Hypoxia Inducible Factor-1 Regulated Cytokines
}

\author{
Savalan Babapoor-Farrokhran ${ }^{1}$, Deanna Gill ${ }^{2}$, and Sumeet Mainigi ${ }^{1}$ \\ ${ }^{1}$ Albert Einstein Medical Center \\ ${ }^{2}$ Emory University Hospital
}

June 23, 2020

\begin{abstract}
Atrial fibrillation (AF) is a common arrhythmia that has major morbidity and mortality. Hypoxia plays an important role in AF initiation and maintenance. Hypoxia inducible factor (HIF), the master regulator of oxygen homeostasis in cells, plays a fundamental role in the regulation of multiple chemokines and cytokines that are involved in different physiological and pathophysiological pathways. HIF is also involved in the pathophysiology of AF induction and propogation mostly through structural remodeling such as fibrosis, however some of the cytokines discussed have even been implicated in electrical remodeling of the atria. In this article, we highlight the association between HIF and some of its related cytokines with AF. Additionally, we provide an overview of the potential diagnostic benefits of using the mentioned cytokines as AF biomarkers. Research discussed in this review suggests that the expression of these cytokines may correlate with patients who are at an increased risk of devleoping AF. Furthermore, cytokines that are elevated in patients with AF can assist clinicians in the diagnosis of suspect paroxysmal AF patients. Interestingly, some of the cytokines have been elevated specifically when AF is associated with a hypercoaguable state, suggeting that they could be helpful in the clinician's and patient's decision to begin anticoagulation. Finally, more recent research has demonstrated the promise of targeting these cytokines for the treatment of AF. While still in its early stages, tools such as neutralizing antibodies have proved to be efficacious in targetting the HIF pathway and treating or preventing AF.
\end{abstract}

\section{Keywords}

Atrial fibrillation, Cytokines, Hypoxia Inducible Factor-1, Vascular Endothelial Growth Factor, Plasminogen activator inhibitor-1, Platelet-derived growth factor, Insulin-Like Growth Factor Binding Protein-3, InsulinLike Growth Factor-1.

\section{Introduction}

Atrial fibrillation (AF) is the most common clinically significant arrhythmia, and it is responsible for major morbidity and mortality. ${ }^{1}$ There are four fundamental pathophysiological mechanisms of AF including: electrical remodeling, structural remodeling, autonomic nervous system changes, and Ca2+ handling abnormalities. ${ }^{2,3,4}$ Atrial fibrosis is the most widely studied and is considered a key factor leading to the development of AF. While evidence linking AF and atrial fibrosis is substantial, it is less clear why atrial fibrosis develops. Hypoxia is hypothesized to play a major role in the pathogenesis of fibrosis. With continued atrial fibrosis, structural remodeling becomes irreversible and leads to sustained AF. ${ }^{5}$ Chronic hypoxia alters $\mathrm{Ca}^{2+}$ handling in rat cardiomyocytes, changes the expression of gap junction proteins such as connexins in mice, and induces angiotensin II production with downstream atrial fibrosis in rats and causes electrical remodeling in patients with obstructive sleep apnea (OSA) ${ }^{6,7,8,9}$ AF consumes more energy compared to non-fibrillatory states. The high energy consumption of atrial excitation leads to tissue ischemia during AF, 
creating a cycle of tissue injury and remodeling. ${ }^{10}$ Lammers et al. reported that hypoxia causes inhomogeneous refractory periods, decreased conduction velocity and wavelength, increased conduction heterogeneity, and reentrant arrhythmias in isolated superfused rabbit left atrial preparations. ${ }^{11}$

While AF can perpetuate hypoxia induced changes due to its increased oxygen demand, hypoxia is also a likely proponent in the initial development of AF for many patients. For example, OSA and nocturnal hypoxia are known to increase the rate of $\mathrm{AF}$ development. In fact the prevalence of OSA in patients with AF ranges from 32\%-49\%. ${ }^{12}$ OSA and chronic hypoxia have roles in the development of AF ranging from altered metabolic enzyme expression to disturbances in the autonomic nervous system to structural remodeling through atrial fibrosis. ${ }^{13}$ Gramley et al. studied human cardiac allograft recipients over a 10 year period and found that not only did cardiac fibrosis follow periods of hypoxia as determined by increased levels of hypoxia inducible factor-1 (HIF1), but that the resulting tissue fibrosis may have contributed to worsening hypoxia by increasing oxygen's diffusion distance. ${ }^{14}$ In addition to increasing the diffusion distance, Colucci et al. describes how cardiac remodeling and fibrosis could impair coronary microcirculation causing ischemia and decrease the transport of oxygen and other nutrients. ${ }^{15,16}$ Interestingly, even without evidence of fibrosis atrial ischemia and hypoxia promotes AF in dog animal models by causing local conduction slowing and promoting reentry. ${ }^{17}$ In this review, we set out to highlight important cytokines involved in the pathophysiology of AF and are regulated by HIF.

\section{HIF and Atrial Fibrillation}

Hypoxia activates multiple pathways. One of the most studied pathways is regulated by a transcription factor HIF-1. HIF-1 senses and responds to oxygen levels ensuring oxygen homeostasis. Specifically, when the cell is experiencing hypoxic conditions both the $\alpha$ and $\beta$ subunits of HIF-1 bind together and localize to the nucleus where the heterodimer signals for changes in gene expression. ${ }^{18}$ The gene targets of the aforementioned HIF-1 response play roles in processes such as: regulating inflammation, angiogenesis, oxygen supply, cell proliferation, cell renewal, epithelial to mesenchymal transition, metastasis and invasion, redox homeostasis, apoptosis, and adaptive survival mechanisms. ${ }^{19}$ The HIF- $1 \alpha$ subunit in particular has been studied in the cardiomyocytes response to hypoxia and it has been linked to the development of AF. ${ }^{20,21,22}$ It has been well established that OSA causes intermittent hypoxia, which as mentioned above affects a large number of patients, especially in America. The intermittent hypoxia experienced has been associated with the activation with HIF-1 in tumors, such as colorectal cancer cells. ${ }^{23}$ It is reasonable to assume that a similar activation pathway occurs in cardiac myocytes, giving the association between AF and OSA mentioned above. Thijssen et. al, reported an increase of HIF-1 $\alpha$ expression in the response of goat cardiomyocytes to AF. ${ }^{21}$ In the study by, Ogi et. al, HIF-1 $\alpha$ levels were elevated in patients with AF, further they hypothesized that the eventual structural remodeling was secondary to myocardial hypoxia. ${ }^{24}$ Interestingly, HIF-1 has been detected specifically in peri-left atrial adipose and subsequently shown to be involved in fibrotic remodeling generating a substrate for $\mathrm{AF} .{ }^{25} \mathrm{In}$ a study by Xu et al. left atrial samples from patients with permanent, persistent, and paroxysmal AF were compared to patients in sinus rhythm. They found that patients who had permanent or persistent AF had increased expression of HIF1- $\alpha$ from left atrial biopsies than patients with paroxysmal AF or patients in sinus rhythm suggesting that HIF1- $\alpha$ plays a strong role in the structural remodeling, which underlies AF initiation and propagation. ${ }^{26}$ There have been dozens of HIF target genes identified. These HIF targets have roles in multiple physiological and pathophysiological pathways that are mentioned above. Today, this number is still increasing. ${ }^{27,28}$

\section{Vascular Endothelial Growth Factor}

Vascular endothelial growth factor (VEGF) is a major angiogenic and inflammatory cytokine, which is produced by located in ischemic tissues and growing tumors. It mediates numerous biological processes including vasculogenesis, vascular permeability, cell migration, survival, proliferation, and differentiation. ${ }^{29}$

There are 4 VEGF sub-types we will focus on for this review: A, B, C, and D. They bind to 3 types of VEGF-receptors (VEGF-R): 1, 2, and 3. ${ }^{30}$

Expression and upregulation of VEGF is induced in cells exposed to ischemia, hypoxia, and oxidative stress. ${ }^{29}$ 
HIF-1 has been shown to play a major role in the up regulation of VEGF. ${ }^{31}$ It has been demonstrated that in atrial samples obtained from AF patients, HIF-1 $\alpha$ protein, VEGF mRNA, VEGF protein, and VEGFR-1 mRNA were upregulated compared to patients with normal sinus rhythm. ${ }^{22}$ In addition, increased VEGF-D concentrations were associated with AF and specifically VEGF is highly expressed within the pulmonary veins-atrial junction, the main induction site for AF. ${ }^{32,33}$ A number of triggers have been studied as the cause of increased VEGF release including mechanical stretch, ischemia and inflammation; ${ }^{34,35,36}$ these triggers will be discussed below.

Kim et al. demonstrated that myocardial mechanical stress enhances HIF-1 nuclear localization and subsequent VEGF upregulation in the rat myocardium. ${ }^{37}$ Similarly, Li et al. demonstrated that VEGF mRNA expression is increased during myocardial stretch in vivo by inflation of an intraventricular balloon in the rat heart. The magnitude of end diastolic pressures generated during this study were equivalent to those experienced in conditions such as congestive heart failure, myocardial infarction, and long-standing hypertension, as well as valvular diseases. Their group chose this method over the myocardial infarction model in order to see if VEGF would be triggered purely based on mechanical stress without the ischemic and inflammatory pathways. ${ }^{38}$ Seko et al. also reported that cultured rat cardiac myocytes upregulated VEGF mRNA and secreted VEGF under pulsatile mechanical stretch in vitro. ${ }^{39}$ In another study by Seko et al, they investigated serum levels of VEGF in patients with AF before and after defibrillation and demonstrated that VEGF levels along with TGF- $\beta 1$ levels decreased significantly after defibrillation suggesting that tachycardia induced mechanical overload could increase VEGF levels. ${ }^{40}$ Further, mechanical overload leads to atrial stretch and remodeling which has a close relationship in the development of AF. ${ }^{41}$ Scridon et. al demonstrated that patients with paroxysmal AF had high peripheral and left atrial, but similar pulmonary vein levels of VEGF compared with non-AF controls. This evidence suggests that the left atrium itself is the source of the elevated VEGF in patients with paroxysmal AF. Interestingly, this paper mentions the strong correlation between mechanical stretch and VEGF levels mentioned above and suggests it as the reason for VEGF only being upregulated in paroxysmal AF patients and not persistent AF patients. They reason that because of the fibrotic changes from long standing AF, mechanical stress on the atrial walls are decreased leading to lower VEGF levels. ${ }^{36}$ These studies all suggest that mechanical overload can upregulate VEGF secretion by cardiac myocytes, specifically those in the left atrium.

Situations and diseases that promote cardiac ischemia and inflammation such as cardiac surgery and coronary artery disease, respectively, increase a patient's propensity for developing AF. In fact, in a study by Granier et al. it was discovered that patients who received corticosteroids before a cardiac operation had a decreased likelihood of developing AF ${ }^{42}$ While inflammation is known to promote fibrosis and thereby AF, Serban et al. postulated it also affected the electrophysiological properties of the myocardium. The group found that in patients who had stable coronary artery disease (CAD), chronic inflammation and ischemia are associated with pro-arrhythmic atrial electrical remodeling. Higher VEGF levels, used as a marker of ischemia, were found in the right atrial appendage of patients who were undergoing coronary artery bypass and were associated with an increase in the duration of atrial depolarization and a decrease in the velocity of atrial depolarization. ${ }^{43}$ In addition, it has been shown that VEGF increases vascular permeability and promotes edema, which can affect the electrophysiology of the heart. VEGF-induced cardiac edema results in disruption of intercalated disc's nano-domains that in turn facilitates arrhythmias. In the study by Mezache et al, they suggest that disrupting intercalated disc's nanodomains by VEGF-A-induced vascular leak elevates AF risk by slowing conduction. ${ }^{44}$

In addition to VEGF's effects on the development of AF, other associations with VEGF and AF have been made, including VEGF's role in the development of hypercoagulable states. Choudhury et al. reported that AF patients have higher levels of VEGF compared to control patients and its plasma levels correlate with the level of soluble CD40 ligand. They propose that increased VEGF levels and other angiogenic factors and their interplay with platelet activation via CD40 ligand contributes to the prothrombotic state in AF. ${ }^{45}$ In the study by Chung et al, they analyzed plasma levels of VEGF, tissue factor (TF) and sFLT-1 in AF and control patients. They found that chronic AF was associated with increased VEGF and TF levels. They further reported that there is a significant relationship between VEGF and sFLT-1levels with TF levels and 
that is contributing to hypercoagulable state found in AF. ${ }^{46}$ In a similar study, high levels of VEGF in AF patients was proposed to be contributing to prothrombotic states in $\mathrm{AF}^{47}$

\section{Plasminogen activator inhibitor-1}

Plasminogen activator inhibitor-1 (PAI-1), inhibits both types of plasminogen activator, tissue and urokinase (t-PA and u-PA). PAI-1 is involved with cardiovascular disease mainly as an inhibitor to t-PA, whereas its role with u-PA is more implicated in cancer. ${ }^{48}$ PAI-1 is synthesized by many different cell types including cardiomyocytes, adipocytes, vascular endothelial cells, macrophages, and fibroblasts. Subsequently, due to it's varied expression, PAI has been linked to fibrosis in various organs including the kidneys, liver, and heart. ${ }^{48}$ Importantly, it has been established that under hypoxic conditions, the expression of PAI-1 is mainly controlled by HIF-1. ${ }^{49,50}$

It has been shown that mice deficient in PAI-1 have increased levels of t-PA and u-PA leading to inflammation and infiltration of macrophages with subsequent fibrosis. Further, a study by De With et al. demonstrated in a 392 patient, observational, prospective study, that lower levels of PAI-1 were associated with a higher incidence of $\mathrm{AF}$ progression (i.e. paroxysmal $\mathrm{AF}$ transcending to persistent or permanent AF.). ${ }^{51}$ The group hypothesized that lower levels of PAI-1 were associated with higher rates of fibrinolysis due to disinhibition of t-PA and u-PA leading to increased levels of fibrin degradation products which serve as chemokines for inflammatory responders such as macrophages. The inflammation therefore leads to fibrosis, which begets AF. ${ }^{51}$ PAI-1 has also been evaluated in patients specifically with paroxysmal AF. Negreva et al. reported that PAI-1 activity is lower in patients with paroxysmal AF compared with control patients suggesting that there is high plasma fibrinolytic activity even during the first 24 hours of the disease. ${ }^{52}$ Gramley et al. observed that with increasing duration of AF, PAI-1 expression decreases, which they attributed to increased atrial fibrosis. ${ }^{53}$ Years before even hypothesizing of mechanism for AF's relationship with PAI-1, Pretorius et al. reported that perioperative PAI-1 levels can be predictive of developing postoperative AF in patients undergoing coronary artery bypass surgery. ${ }^{54}$ However, conversely to what De With et al. ${ }^{51}$ and Negreva et al. found, PAI-1 levels were noted to be lower in patients with sinus rhythm and higher levels of PAI were associated with an increased risk of $\mathrm{AF}$ development after surgeries requiring cardiopulmonary bypass surgery. ${ }^{54}$ Similarly, Tveit et al. showed that levels of PAI-1 were predictive of AF recurrence after electrical cardioversion. Like Pretorius et al. they found that higher levels of PAI-1 were associated with higher AF recurrence rates after cardioversion. ${ }^{55}$ In a recent cohort study by Mulder et al., PAI-1 and t-PA levels were elevated in AF patients. However, in their study and contrary to previous findings, there was no association between PAI-1 levels and the onset of AF. ${ }^{56}$

Interestingly, groups that have looked at the relationship between PAI-1 and the hypercoagulable state of AF have found more consistent results. First, it has been shown that PAI-1 levels and markers of endothelial dysfunction have a significant positive correlation with left atrial volume. ${ }^{57}$ Further, a study by Otto et al. assessed the fibrinolytic deficit of patients with AF by checking levels of PAI-1 in patients with AF prior to ablation. They demonstrated that PAI-1 levels were significantly elevated at baseline and decreased levels were observed 1 month post ablation. ${ }^{58}$ However, despite these findings, Liles et al. reported elevated levels of PAI-1 in AF patients even after receiving oral anticoagulation. ${ }^{59}$ Similarly, in a study by Berge et al. AF was independently associated with higher levels of PAI-1 activity. ${ }^{60}$ In a canine model of AF the expression of the PAI-1 was increased in the left atrium along with significant elevation of plasma levels. This was along with decreased endocardial NOS expression and NO concentration suggesting thrombosis in $\mathrm{AF} .{ }^{61}$

\section{Platelet-derived growth factor}

Platelet-derived growth factor (PDGF) is a growth factor that plays a significant role in angiogenesis, organogenesis, and mitogenesis. It is also involved in the proliferation of fibroblasts, osteoblasts, tenocytes, vascular smooth muscle cells and mesenchymal stem cells. ${ }^{62}$ It has been shown that hypoxia and HIF-1 $\alpha$ play a major role in the regulation of PDGF activity. ${ }^{63}$ In fact, a study by Chen et al. demonstrated that PDGF signaling is directly related to the Jak/STAT pathway, a known transcriptional regulator of HIF expression. The group even found that inhibition of the Jak/STAT pathway reduced fibrosis in canine fibroblasts in vitro 
and attenuated fibrosis of post myocardial infarction mice. ${ }^{64}$ There are four types of PDGF, A through D, and two types of PDGF receptors, PDGFR-alpha and beta. While all of the PDGF subtypes are present normally throughout development and adulthood, altered expression of PDGF-C and PDGF-D, for example, have been shown to cause significant cardiac fibrosis in mice. ${ }^{65,66}$ In fact, many studies in mice and rats have been suggestive that PDGF plays an important role in the development of atrial fibrosis and AF via NFkB signaling and TGF- $\beta$ activation. ${ }^{67,68}$ More recently, Jiang et al. has shown that PDGF-B/PDGFR-beta signaling are selectively upregulated in the atria of canine heart failure models. Differential expression of PDGF in the atria is important for atrial-selective fibrosis, a hallmark of AF. To further understand the relationship between PDGF-B/PDGFR- $\beta$ signaling and atrial fibrosis, they studied its expression in the myocardium of patients with chronic AF versus patients in sinus rhythm by collecting right atrial samples from patients who were undergoing cardiac surgery. Concurrently, they studied the relationship between PDGF-B/PDGFR- $\beta$ signaling and AF in canines that were in AF for either 1, 2 or, 4 weeks. In the patients with AF, an increased level of PDGF and PDGFR- $\beta$ proteins were found in the myocardium. At the same time, they found that not only were levels of PDGF-B and PDGFR- $\beta$ increased in the canine models with $\mathrm{AF}$, but the levels continued to increase as the time spent in AF increased so that the canines in the 4 week group had higher levels of PDGF-B and PDGFR- $\beta$ than the canines in the 1 week or 2 week group. In addition, they found that the increasing levels of collagen deposition, analyzed by trichrome staining, tracked with the increase in PDGFR- $\beta$ specifically. Although the evidence surrounding PDGF and atrial fibrosis is more robust, studies have also shown that PDGF has a direct impact on the electrophysiologic properties of myocytes. ${ }^{69}$ As Fiset et al. points out, myofibroblasts are not typically encountered in atrial tissue, and their presence has been associated with increased levels of PDGF, which was thought to only exert only fibrotic effects ${ }^{70}$ until a paper published by Musa et al. described how inhibition of PDGF-AB prevented electrophysiologic remodeling. In the study, myocytes and myofibroblasts from sheep atria were co-cultured and they found a decrease in L-type calcium current density and shortening of the action potential, both of which are known hallmarks of AF development. In addition, they found that these effects were reversed by pretreating the cells with PDGF-AB neutralizing antibody. The same trend was noted when harvested atrial myocytes were exposed directly to PDGF-AB alone and then with the neutralizing antibody. ${ }^{70,71}$ Taken all together, HIF- $1 \alpha$ regulates the fibrogenic cytokine, PDGF, which has been substantially proven to be an effector in the development of atrial fibrosis and possibly electrophysiologic changes, both of which are promoters of $\mathrm{AF}$.

\section{Insulin-Like Growth Factor Binding Protein-3 and Insulin-Like Growth Factor-1}

Insulin-Like Growth Factor Binding Protein-3 (IGFBP-3) is the main Insulin-Like Growth Factor-1 (IGF-1) carrier protein. Felser et al. showed that cultured cells exposed to IGF-1 upregulate HIF-1 $\alpha$ expression and HIF- $1 \alpha$ was proven to be essential for the expression of IGFBP-3. This study demonstrated HIF's lesser known role in energy and glucose metabolism when compared to its established role in oxygen homeostasis. ${ }^{72}$ Additional studies have suggested that IGF-1 is cardioprotective and low levels of IGF have been associated with increased incidence of coronary artery disease and cardiovascular mortality. ${ }^{73}$ In a study by Busch et al. 3160 patients were medically evaluated with a physical exam and a standard electrocardiographic assessment and had IGF-1 and IGFBP-3 serum levels drawn. The patients were followed for a median of 5.2 years and the patients were monitored for the development of AF. They found that patients with low levels of IGF-1 and low ratios of IGF-1:IGFBP-3 had an increased likelihood of developing AF. ${ }^{74}$ Their study is consistent with the previous notion that IGF-1 levels are cardioprotective. Several studies have cited reasons for this inverse relationship including: inflammation secondary to endothelial cell dysfunction, stress and hypoxia response via the mechanistic Target of Rapamycin (mTOR) pathway, antiarrhythmic properties, and the development of fibrosis. ${ }^{75,76,77,78}$ Wang et al. specifically analyzed the effects of IGF-1 on atrial fibrosis in rats and in contrast to the previous study, they found that IGF-1 plays a pro-fibrotic role, and in rats with IGF-1 silencing AF inducibility was ameliorated. ${ }^{79}$ Namely, Gonzalez-Guerra et al. demonstrated that IGF-1 deficiency was associated with a decrease in heart contractility as well as interstitial and perivascular fibrosis. Not only were mice hearts with decreased IGF-1 levels shown to be fibrotic, but the fibrotic changes were able to be reversed by supplementing the mice with IGF-1. Interestingly, the hearts that were IGF-1 deficient 
and not treated with IGF-1 supplement overexpressed Serpine1, also known as PAI-1, mentioned above. The IGF-1 deficient mice that demonstrated increased Serpine1 levels were exquisitely sensitive to IGF-1 replacement. This pattern of descriptive, non-specific, and at times contradictory evidence surrounding IGF1 and IGFBP-3 as they pertain to AF development can be convoluted. However, they all confirm the idea that IGF-1 and IGFBP-3 have a role in cardiovascular disease, including AF, and is worth studying in more detail.

\section{Potential for Diagnostic and/or Therapeutic Targets}

There are multiple underlying molecular mechanisms contributing to AF. Targeting these pathways utilizing different safer approaches, e.g. at the level of gene expression, ${ }^{80,81}$ have been suggested for AF treatment and prevention. Hypoxia is a known cause for the development of AF and as a direct respondent to changing oxygen levels, HIF has suggested involvement in AF physiology and pathophysiology. There has been a significant increase in the number of identified target genes that HIF regulates. Blocking HIF and proteins that it regulates have been studied as therapeutic strategies in many diseases. Some of these genes and their coded cytokines have been implicated in the development and progression of AF. The potential for targeting these cytokines as a means for preventing the development and/or progression of AF and the role of these cytokines as a potential biomarker and early diagnostic marker is still in its infancy. Wang et al. demonstrated how the different sub-types of VEGF and VEGFR act independently in AF patients. For instance, VEGF-A and VEGFR-1 levels were increased in AF, whereas VEGFR-2 levels were lower in the AF group when compared to the control group. This finding emphasized the individual roles of the VEGF and VEGF-R members. Regardless of their positive or negative correlation with AF, this research highlighted these cytokines and cytokine receptors as possible therapeutic and biomarker targets for AF. ${ }^{82}$ In an interesting study by Woitek et al. the authors demonstrated the cytoprotective properties of VEGF-B in a canine model of tachycardia induced dilated cardiomyopathy. They noted that the production of reactive oxygen species was significantly lower in tissue obtained from paced hearts that had been transduced with VEGF-B suggesting a safe and efficacious treatment strategy for dilated cardiomyopathy. ${ }^{83}$ In a similar study by Pepe et al. intramyocardial injection of VEGF-B gene delivery delayed the progression of dilated cardiomyopathy. ${ }^{84}$ Even though these studies are promising, more studies need to be done in atrial fibrillation to delineate possible therapeutic effects of promoting certain subtypes of VEGF or inhibiting them. Tofler and colleagues demonstrated that PAI-1 levels predict first cardiovascular disease events in Framingham Heart Study. ${ }^{85}$ De With et al. analyzed PAI-1 plasma levels as a factor for AF progression. ${ }^{51}$ Pretorius et al. demonstrated PAI-1 as a predictor and biomarker of postoperative atrial fibrillation after cardiopulmonary bypass. ${ }^{54}$ Targeting PDGF has been studied for AF treatment as well. ${ }^{70}$ As discussed previously, Musa et al. demonstrated that the inhibition of PDGF-AB signaling prevents the electromechanical remodeling of adult atrial myocytes. And they suggest that PDGF may serve as a potential therapeutic target for AF. Additionally, Busch and colleagues reported that low IGF-1:IGFBP-3 coincide with an increased incidence of AF that makes these two target suitable biomarkers of AF. ${ }^{74}$ Given the support for many of these cytokines as biomarkers for AF, they should also be considered as diagnostic tools and not just potential treatment targets. The suspected paroxysmal AF patient can be a diagnostic challenge, and beginning anticoagulation is not without risk. These cytokines offer a promising diagnostic tool for these patients, especially considering that some of them, VEGF ${ }^{46,47}$ for example have been shown to have differentiated expression that correlates to the hypercoaguable state of AF patients.

\section{Gaps in our current knowledge}

The pathophysiological mechanisms of AF are complex and some of them are not well understood. Although recent studies have provided insight on the mechanisms and interactions of these cytokines and illustrated how they are related to HIF and AF, much remains to be elucidated. For example, multiple cytokines that HIF regulates seem to have opposing effects in different studies. For example, high PAI-1 levels were associated with sinus rhythm in some studies, but with AF in other studies and the reason for this discrepancy is not clear. ${ }^{51,54}$ Additionally, while molecular targeting of these cytokines appears to be a safe and efficacious treatment for some disease processes such as dilated cardiomyopathy, ${ }^{83}$ the possibility of them being treatment 
targets for AF remains hypothetical. Further studies are needed to clarify mechanisms and interactions of these cytokines and their effect on pathophysiologic processes in order to identify pharmacological targets for treatment of $\mathrm{AF}$ and markers of risk for the development of $\mathrm{AF}$.

\section{Discussion}

Specifically, we selected VEGF, PAI-1, PDGF, IGF-1, and IGFBP-3 as model cytokines for this review because of the strong evidence that links them to both HIF and AF. These cytokines perpetuate either the development or propagation of AF through a variety of mechanisms, but most commonly they end with the promotion of atrial fibrosis and occasionally electrophysiologic remodeling. Multiple studies were done linking VEGF with HIF and AF through fibrotic and electrical changes; they were subdivided into groups that focused on triggers for VEGF upregulation including mechanical stretch, ischemia, and inflammation. ${ }^{34,35,36}$ PAI-1 mediates AF primarily through its role in fibrosis and while the studies investigating its role have mixed results, investigators were able to use PAI- 1 levels to predict who was at higher risk of developing AF. ${ }^{51,54,55}$ Similarly to VEGF, PDGF was shown to have roles in both fibrotic and electrical changes leading to AF; further research demonstrated that inhibition of PDGF with a neutralizing antibody decreased the rate of AF development. ${ }^{69,70,71}$ Finally IGF-1 and IGFBP-3 were shown to play a fibrotic role, but additional research suggested that supplementation of IGF in already fibrotic hearts could reverse the fibrosis and diminish the increased risk of developing AF. ${ }^{78}$ While many of the mentioned studies focus on the associations between these cytokines, HIF, and AF, an increasing amount of research has been focusing on how levels of the cytokines can predict which patients are more likely to develop AF and how inhibition of these cytokines can prevent the development of AF. ${ }^{54,55,71,78}$ These studies emphasize the importance of these cytokines as potential biomarkers and/or therapeutic targets. ${ }^{64,65}$ Therefore, HIF and the proteins that it regulates could be promising targets for future therapy and a practical tool to predict AF development in high risk patients. All these studies have a grand impact on our understanding of the mechanisms involved in the up regulation and downstream effect of these proteins, however literature lacks studies that explore therapeutic benefits of these agents and more work needs to be done in that regard.

\section{References}

1. Kannel WB, Wolf PA, Benjamin EJ, Levy D. Prevalence, incidence, prognosis, and predisposing conditions for atrial fibrillation: population-based estimates. Am J Cardiol. 1998;82 , 2-9.

2. Nattel S, Harada M. Atrial Remodeling and Atrial Fibrillation: Recent Advances and Translational Perspectives. J Am Coll Cardiol.2014;63, 2335-2345.

3. Wakili R, Voigt N, Kaab S, Dobrev D, Nattel S. Recent advances in the molecular pathophysiology of atrial fibrillation. J Clin Invest . 2011;121 , 2955-2968.

4. Nattel S, Dobrev D. The multidimensional role of calcium in atrial fibrillation pathophysiology: mechanistic insights and therapeutic opportunities. Eur Heart $J$. 2012;33 1870-1877.

5. Shinagawa K, Shi YF, Tardif JC, Leung TK, Nattel S. Dynamic nature of atrial fibrillation substrate during development and reversal of heart failure in dogs. Circulation, 2002;105 , 2672-2678.

6. Pei JM, Kravtsov GM, Wu S, Das R, Fung ML, Wong TM. Calcium homeostasis in rat cardiomyocytes during chronic hypoxia: a time course study. Am J Physiol Cell Physiol.2003;285,1420-1428.

7. Gemel J, Su Z, Gileles-Hillel A, Khalyfa A, Gozal D, Beyer EC. Intermittent hypoxia causes NOX2dependent remodeling of atrial connexins. BMC Cell Biol. 2017;18 .

8. Su F, Zhang W, Chen Y, Ma L, Zhang H, Wang F. Significance of hypoxia-inducible factor-1 $\alpha$ expression with atrial fibrosis in rats induced with isoproterenol. Exp Ther Med . 2014;8 , 1677-1682.

9. Dimitri H, Ng M, Brooks, A. G. Kuklik et al. Atrial remodeling in obstructive sleep apnea: Implications for atrial fibrillation. Heart Rhythm. 2012;9, 321-327. 
10. White CW, Kerber RE, Weiss HR, Marcus ML. The effects of atrial fibrillation on atrial pressure-volume and flow relationships. Circ Res. 1982;51, $205-215$.

11. Lammers WJ, Kirchhof CH, Bonke FI, Allessie MA. Vulnerability of rabbit atrium to reentry by hypoxia. Role of inhomogeneity in conduction and wavelength. Am J Physiol. 1992;262, 47-55.

12. Todd K, McIntyre W, Baranchuk A. Obstructive sleep apnea and atrial fibrillation. Nat Sci Sleep . 2010;2, 39-45.

13. Channaveerappa D. Atrial electrophysiological and molecular remodelling induced by obstructive sleep apnea. J Cell Mol Med . 2017;21 , 2223-2235.

14. Gramley F, Lorenzen J, Pezzella, et al. Hypoxia and myocardial remodeling in human cardiac allografts: a time-course study. J Heart Lung Transplant . 2009;28 , 1119-26.

15. Colucci W. Molecular and Cellular Mechanisms of Myocardial Failure.The American Journal of Cardiology. 1987;80 , 15-25.

16. De Boer RA, Pinto YM, Van Veldhuisen DJ. The imbalance between oxygen demand and supply as a potential mechanism in the pathophysiology of heart failure: the role of microvascular growth and abnormalities Microcirculation . 2003;10 , 113-126.

17. Sinno H, Derakhchan K, Libersan D, Merhi Y, Leung TK, Nattel S. Atrial ischemia promotes atrial fibrillation in dogs Circulation.2003;107, 1930-1936.

18. Semenza GL. Hypoxia-inducible factor 1 (HIF-1) pathway.Science's STKE. 2007;407 .

19. Semenza GL. Hypoxia-inducible factors in physiology and medicine. Cell. 2012;148 , 399-408.

20. Semenza GL. Hypoxia-inducible factor 1 and cardiovascular disease.Annu Rev Physiol. 2014;76 , 39-56.

21. Thijssen VL, Van der Velden HM, Van Ankeren EP, et al. Analysis of altered gene expression during sustained atrial fibrillation in the goat. Cardiovasc Res . 2002;54, 427-437.

22. Gramley F, Lorenzen J, Jedamzik B, et al. Atrial fibrillation is associated with cardiac hypoxia. Cardiovasc Pathol.2010;19, 102-111.

23. Martinez CA, Kerr B, Jin C, Cistulli PA, Cook KM. Obstructive sleep apnea activates HIF-1 in a hypoxia dose-dependent manner in HCT116 colorectal carcinoma cells. Int J Mol Sci. 2019;20 , 445.

24. Ogi H, Nakano Y, Nilda S et al. Is structural remodeling of fibrillated atria the consequence of tissue hypoxia? Circ J . 2010;74, 1815-1821.

25. Abe I, Teshima Y, Kondo H, et al. Association of fibrotic remodeling and cytokines/chemokines content in epicardial adipose tissue with atrial myocardial fibrosis in patients with atrial fibrillation. Heart Rhythm . 2018;15, 1717-1727.

26. Xu Y, Sharma D, Du F, Liu Y. The role of Toll-like receptor 2 and hypoxia-induced transcription factor- $1 \alpha$ in the atrial structural remodeling of non-valvular atrial fibrillation. Int J Cardiol.2013;168 , 2940-2941.

27. Dengler VL, Galbraith MD, Espinosa JM. Transcriptional regulation by hypoxia inducible factors. Crit Rev Biochem Mol Biol . 2014;49, 1-5.

28. Semenza G. Targeting HIF-1 for cancer therapy. Nat Rev Cancer. 2003;3 , 721-732.

29. Ferrara N, Houck K, Jakeman LY, Leung DW. Molecular and biological properties of the vascular endothelial growth factor family of proteins. Endocrine reviews. 1992;13 , 18-32.

30. Stuttfeld E, Ballmer-Hofer K. Structure and function of VEGF receptors. IUBMB life. 2009;61, 915-922. 
31. Forsythe JA, Jiang BH, Iyer NV et al. Activation of vascular endothelial growth factor gene transcription by hypoxia-inducible factor 1. Mol Cell Biol. 1996;16 , 4604-4613.

32. Berntsson J, Smith JG, Johnson LSB, et al. Increased vascular endothelial growth factor D is associated with atrial fibrillation and ischaemic stroke. Heart. 2019;105, 553-558.

33. Iwasaki YK, Yamashita T, Sekiguchi A, Hayami N, Shimizu W. Importance of pulmonary vein preferential fibrosis for atrial fibrillation promotion in hypertensive rat hearts. Can J Cardiol.2016;32, 767-776.

34. Yao C, Veleva T, Scott L, et al. Enhanced cardiomyocyte NLRP3 inflammasome signaling promotes atrial fibrillation. Circulation.2018;138, 2227-2242.

35. Li J, Solus J, Chen Q, Rho YH, Milne G, Stein CM, Darbar D. Role of inflammation and oxidative stress in atrial fibrillation. Heart Rhythm . 2010;7 , 438-444.

36. Scridon A, Morel E, Nonin-Babary E, Girerd N, Fernandez C, Chevalier P. Increased intracardiac vascular endothelial growth factor levels in patients with paroxysmal, but not persistent atrial fibrillation. Europace. 2012;14, 948-953.

37. Kim CH, Cho YS, Chun YS, Park JW, Kim MS. Early expression of myocardial HIF-1 $\alpha$ in response to mechanical stresses: regulation by stretch-activated channels and the phosphatidylinositol 3-kinase signaling pathway. Circulation. 2002;90, 25-33.

38. Li J, Hampton T, Morgan JP, Simons M. Stretch-induced VEGF expression in the heart. J Clin Invest. 1997;100, 18-24.

39. Seko Y, Takahashi N, Shibuya M, Yazaki Y. Pulsatile stretch stimulates vascular endothelial growth factor (VEGF) secretion by cultured rat cardiac myocytes. Biochem Biophys Res Commun.1999;254 , 462465.

40. Seko Y, Nishimura H, Takahashi N, Ashida T, Nagai R. Serum Levels of Vascular Endothelial Growth Factor and Transforming Growth Factor-b1 in Patients with Atrial Fibrillation Undergoing Defibrillation Therapy. Japanese heart journal. 2000;41, 27-32.

41. De Jong A, Maass AH, Oberdorf-Maass SU, et al. Cyclical stretch induces structural changes in atrial myocytes. J Cell Mol Med . 2013;17 , 743-753.

42. Granier M, Massin F, Pasquie JL. Pro- and Anti-Arrhythmic Effects of Anti-Inflammatory Drugs. Anti-Inflamm AntiAllergy Agents Med Chem. 2013;12, 83-93.

43. Serban RC, Balan AI, Perian M, et al. Atrial electrical remodeling induced by chronic ischemia and inflammation in patients with stable coronary artery disease. Chin J Physiol . 2019;62 , 11.

44. Mezache L, Struckman H, Greer-Short A, et al. Vegf-Induced Vascular Leak Promotes Atrial Fibrillation by Disrupting Intercalated Disc Nanodomains. Biophys J . 2019;116 , 32a.

45. Choudhury A, Freestone B, Patel J, Lip GY. Relationship of soluble CD40 ligand to vascular endothelial growth factor, angiopoietins, and tissue factor in atrial fibrillation: a link among platelet activation, angiogenesis, and thrombosis? Chest . 2007;132, 1913-1919.

46. Chung NA, Belgore F, Li-Saw-Hee FL, et al. Is the hypercoagulable state in atrial fibrillation mediated by vascular endothelial growth factor? Stroke . 2002;33 , 2187-2191.

47. Freestone B, Chong AY, Lim HS, Blann A, Lip GY. Angiogenic factors in atrial fibrillation: a possible role in thrombogenesis? Ann Med. 2005;37, 365-372.

48. Ghosh AK, Vaughan DE. PAI-1 in tissue fibrosis. J Cell Physiol . 2012;227 , 493-507.

49. Kietzmann T, Jungermann K, Gorlach A. Regulation of the hypoxia-dependent plasminogen activator inhibitor 1 expression by MAP kinases. Thromb Haemost . 2003;89 , 666-673. 
50. Carroll VA, Ashcroft M. Role of hypoxia-inducible factor (HIF)- $1 \alpha$ versus HIF- $2 \alpha$ in the regulation of HIF target genes in response to hypoxia, insulin-like growth factor-I, or loss of von Hippel-Lindau function: implications for targeting the HIF pathway. Cancer res . 2006;66 , 6264-6270.

51. De With RR, Marcos EG, Dudink EA, et al. Atrial fibrillation progression risk factors and associated cardiovascular outcome in well-phenotyped patients: data from the AF-RISK study. Europace.2019;339 .

52. Negreva M, Georgiev S, Vitlianova K. Early effects of paroxysmal atrial fribrillation on plasma markers of fibrinolysis. Medicine . 2016;95 .

53. Gramley F, Lorenzen J, Plisiene J, et al. Decreased plasminogen activator inhibitor and tissue metalloproteinase inhibitor expression may promote increased metalloproteinase activity with increasing duration of human atrial fibrillation. J cardiovasc electrophysiol. 2007;18 , 1076-1082.

54. Pretorius M, Donahue BS, Yu C, Greelish JP, Roden DM, Brown NJ. Plasminogen activator inhibitor-1 as a predictor of postoperative atrial fibrillation after cardiopulmonary bypass. Circulation.2007;116, I-1.

55. Tveit A, Seljeflot I, Grundvold I, Abdelnoor M, Smith P, Arnesen H. Levels of PAI-1 and outcome after electrical cardioversion for atrial fibrillation. Thromb res. 2008;121, 447-453.

56. Mulder BA, Geelhoed B, van der Harst P, et al. Plasminogen activator inhibitor-1 and tissue plasminogen activator and incident AF: Data from the PREVEND study. Int J cardiol. 2018;272 , 208-210.

57. Mondillo S. Correlation between left atrial size, prothrombotic state and markers of endothelial dysfunction in patients with lone chronic nonrheumatic atrial fibrillation. Int $J$ cardiol.2000;75 , 227-232.

58. Otto A, Fareed J, Liles J, et al. Fibrinolytic deficit and platelet activation in atrial fibrillation and their postablation modulation. Clin Appl Thromb Hemost. 2018;24 , 803-807.

59. Liles J, Wanderling C, Syed M, Hoppensteadt D, Fareed J. Increased level of thrombotic biomarkers in patients with atrial fibrillation despite traditional and new anticoagulant therapy. Clin Appl Thromb Hemost . 2016;22, 743-748.

60. Berge T, Ulimoen SR, Enger S, Arnesen H, Seljeflot I, Tveit A. Impact of atrial fibrillation on inflammatory and fibrinolytic variables in the elderly. Scand J Clin Lab Invest. 2013;73, 326-333.

61. Han W, Li WM, Song LY, et al. The experimental study on changes of endothelial nitric oxide synthase and plasminogen activator inhibitor-1 protein in the canine atrial fibrillation model. Zhonghua xin xue guan bing za zhi. 2005;33, 69-72.

62. Andrae J, Gallini R, Betsholtz C. Role of platelet-derived growth factors in physiology and medicine. Genes Dev . 2008;22, 1276-312.

63. Schultz K, Fanburg BL, Beasley D. Hypoxia and hypoxia-inducible factor-1 $\alpha$ promote growth factorinduced proliferation of human vascular smooth muscle cells. Am J Phyiol Heart Circ Physiol.2006;290 , 2528-2534.

64. Chen Y, Surinkaew S, Naud P, et al. JAK-STAT signalling and the atrial fibrillation promoting fibrotic substrate. Cardiovasc. Res. 2017; 113 , 310-320.

65. Pontén A, Folestad EB, Pietras K, Eriksson U. Platelet-derived growth factor D induces cardiac fibrosis and proliferation of vascular smooth muscle cells in heart-specific transgenic mice. Circ Res.2005;97, 10361045 .

66. Pontén A, Li X, Thoren P, Aase K, Sjoblom T, Ostman A, Eriksson U. Transgenic overexpression of platelet-derived growth factor-C in the mouse heart induces cardiac fibrosis, hypertrophy, and dilated cardiomyopathy. Am J Pathol. 2003;163, 673-682.

67. Tuuminen R, Nykanen AI, Krebs R, et al. PDGF-A, -C, and -D but not PDGF-B increase TGF- $\beta 1$ and chronic rejection in rat cardiac allografts.Arterioscler Thromb Vasc Biol. 2009;29 , 691-698. 
68. Yang D, Yuan J, Liu G, et al. Angiotensin receptor blockers and statins could alleviate atrial fibrosis via regulating platelet-derived growth factor/Rac1/nuclear factor-kappa B axis. Int J Med Sci.2013;10 , 812-824.

69. Jiang Z, Zhong G, Wen L et al. The Role of Platelet-Derived Growth Factor-B/Platelet-Derived Growth Factor Receptor- $\beta$ Signaling in Chronic Atrial Fibrillation. Cardiology. 2016; 133 , 244-256.

70. Fiset C. Platelet-derived growth factor: A promising therapeutic target for atrial fibrillation. Heart Rhythm . 2013;10 , 1052-1053.

71. Musa H, Kaur K, O'Connell R, et al. Inhibition of platelet-derived growth factor-AB signaling prevents electromechanical remodeling of adult atrial myocytes that contact myofibroblasts. Heart Rhythm.2013;10 , 1044-1051.

72. Feldser D, Agani F, Iyer NV, Pak B, Semenza GL. Reciprocal positive regulation of hypoxia-inducible factor $1 \alpha$ and insulin-like growth factor 2. Cancer res . 1999;59, 3915-3918.

73. Lee WS, Kim J. Insulin-like growth factor-1 signaling in cardiac aging. Biochim Biophys Acta. 2018; 1864, 1931-1938.

74. Busch M, Kruger A, Gross S, et al. Relation of IGF-1 and IGFBP-3 with prevalent and incident atrial fibrillation in a population-based study. Heart Rhythm . 2019;16 , 1314-1319.

75. Polovina MM, Lip GY, Potpara TS. Endothelial (dys)function in lone atrial fibrillation. Curr Pharm Des . 2014;21 , 622-645.

76. Troncoso R, Ibarra C, Vicencio JM, Jaimovich E, Lavandero S. New insights into IGF-1 signaling in the heart. Trends Endocrinol Metab . 2014;25 , 128-137.

77. Ittermann T, Noord CV, Friedrish N, et al. The association between insulin-like growth factor-I and cardiac repolarization. Growth Horm IGF Res. 2012;22, 1-5.

78. Gonzalez-Guerra JL, Castilla-Cortazar I, Aguirre GA, et al. Partial IGF-1 deficiency is sufficient to reduce heart contractibility, angiotensin II sensibility, and alter gene expression of structural and functional cardiac proteins. PLoS One . 2017;12 , E0181760.

79. Wang J, Li Z, Du J, Li J, Zhang Y, Liu J, Hou Y. The expression profile analysis of atrial mRNA in rats with atrial fibrillation: the role of IGF-1 in atrial fibrosis. BMC Cardiovasc Disord . 2019;19 , 40.

80. Babapoor-Farrokhran S, Gill D, Rasekhi RT. The role of long noncoding RNAs in atrial fibrillation. Heart Rhythm . (2020). Doi:10.1016/j.hrthm.2020.01.015

81. Sirish P, Li N, Timofeyev V, et al. Hammock, B. D. Chiamvimonvat, N. Molecular mechanisms and new treatment paradigm for atrial fibrillation.Circ Arrhythm Electrophysiol . 2016;9, 5 .

82. Wang K, Liu Y, Huang S, et al. Does an imbalance in circulating vascular endothelial growth factors (VEGFs) cause atrial fibrillation in patients with valvular heart disease?. J Thorac Dis . 2019;12, 5509.

83. Woitek F, Zentilin L, Hoffman NE, et al. Intracoronary cytoprotective gene therapy: a study of VEGFB167 in a pre-clinical animal model of dilated cardiomyopathy. J Ame Col Cardiol.2015;66 (2):139-153.

84. Pepe M, Mamdani M, Zentilin L, et al. Intramyocardial VEGF-B167 gene delivery delays the progression towards congestive failure in dogs with pacing-induced dilated cardiomyopathy. Circ Res. 2010;106(12):18931903. doi:10.1161/CIRCRESAHA.110.220855

85. Tofler GH, Massaro J, O'Donnell CJ, et al. Plasminogen activator inhibitor and the risk of cardiovascular disease: The Framingham Heart Study. Thromb res . 2016;140 :30-35.

\section{Hosted file}


HIF Cytokines Schematic.pptx available at https://authorea.com/users/336041/articles/461845atrial-fibrillation-the-role-of-hypoxia-inducible-factor-1-regulated-cytokines 